\title{
The Prospects of Using Meshes in Imediate Implant - Based Breast Reconstructions
}

\author{
CRISTINA IULIA FILIP1, SORIN BERBECE2*, LAURA RADUCU ${ }^{1,3}$, IOAN PETRE FLORESCU1, VALERIU ARDELEANU2, \\ CRISTIAN RADU JECAN ${ }^{1,3}$ \\ ${ }^{1}$ University of Medicine and Pharmacy Carol Davila, 8 Eroii Sanitari Str., 050474, Bucharest, Romania \\ 2 Dunarea de J os University, Faculty of Medicine and Pharmacy, 47 Domneasca Str., 800008, Galati, Romania \\ ${ }^{3}$ Clinical Emergency Hospital Agrippa Ionescu, Department of Plastic and Reconstructive Surgery, 7 Ion Mincu Str.,011356, \\ Bucharest, Romania
}

\begin{abstract}
Breast reconstruction involves two majorconditions: to be oncologically safe and to respect the aesthetic of the reconstructed breast. Moreover, every healthcare system in the world manifests a keen tendency to cut back on medical costs, which influences our surgical techniques and breast reconstruction procedures. The use of biological matrices like the acellular dermal matrix has become an acknowledged alternative in implant-based breast reconstruction, in spite of the many impediments and controversies that surround it. However, these matrices are costly and less attainable as compared to synthetic meshes that are conservative with resources and unyielding to the formation of biofilm bacteria. Accordingly, we decided to research the impact of synthetic meshes in implant-based breast reconstruction.
\end{abstract}

Keywords: immediate breast reconstruction, breast implants, syntethic mesh

Implant-based breast reconstrution is responsible for more than $70 \%$ of the breast reconstructions performed in the United States [1], partially due to the fact that immediate breast reconstruction has recently become a customary procedure for both curative and prophylactic mastectomy whenever this is considered oncologically safe [2].

Implant-based breast reconstructions must include an adequate overlay over the breast implant, which is generally composed of the pectoralis major muscle in the superior and infero-medial quadrant. This commonly leads to an inadequate support of the implant and further complications [2]. In this respect, when the acellular dermal matrix (ADM) came into existence, it was seen as a good solution for dealing with this problem.

ADM is an acellular, cryopreserved matrix, that lacks antigenic stimuli. Its use is intended to prevent extrusion and rupture of the implant while assuring its precise placement in the submuscular pocket of the newly reconstructed breast [3].

In spite of being such a widely used procedure, as local tissues are generally insufficient to cover a breast implant, implant-based breast reconstruction (IBBR) can present with issues like capsular contracture, implant rippling and bottoming out, as well as inadequate control of the implant's pocketand defective expansion of the lower pole [4]. ADM was the first tissue substitute used to complete the traditional subpectoral pocket and better define the inframammary fold while allowing better lower pole expansion and a more natural contour for the reconstructed breast [2]. ADM was considered an option not only for its biomechanical properties like elasticity and resistance [5], but also because it allows cellular ingrowth and revascularisation, resulting in a good integration within the implant's capsule and minimal inflammatory reaction [6]. Results obtained with ADM were favorable, but high costs and long-term complication like infection, seroma, flap necrosis and implant explantation reported by a number of studies urged us to search for better alternatives [7].

Synthethic meshes proved efficient in IBBR due to the good supportand control they provide over the inferior and inferolateral poles of the implant, with good inframmary and lateral fold delineation. Moreover, using a synthetic mesh to complete the subpectoral pocket allows for lower pole expansion and usage of an implant with increased volume, leading to a more natural contour and a better chance to attain symmetry [8]. Furthermore, the assistance of synthethic meshes in most surgical specialties makes these materials higly accessible at reasonable costs, with a well-studied low inflammatory reaction and absentbiofilm formation [7]. On the european market, there are three types of synthetic meshes approved for breast reconstruction, each with different properties: Type A mesh - a partially absorbable one, Type B - an unabsorbable mesh and a rapidly absorbable one - Type $C$ [2].

The Type A mesh, original from Germany is a partially absorbable mesh composed of an absorbable component (PGA caprolactone) which lasts for $90-120$ days after surgery and a non-absorbable component (polypropylene) which remains in place for further support [9]. This mesh has a hydrophilic feature which makes its intraoperative manipulation easier if previously immersed in a saline solution. It has been observed that this mesh assimilates well in the implant's capsule, presents good cellular ingrowth and high biocompatibility, while offering ireplaceable support and coverage to the breast implant [10]. Its structural components offer a tensile strength of $70 \mathrm{~N}$ longitudinally and $40 \mathrm{~N}$ crosswise, at a low weight of $28 \mathrm{~g} / \mathrm{m}^{2}$ once the absorbtion process is completed [2].

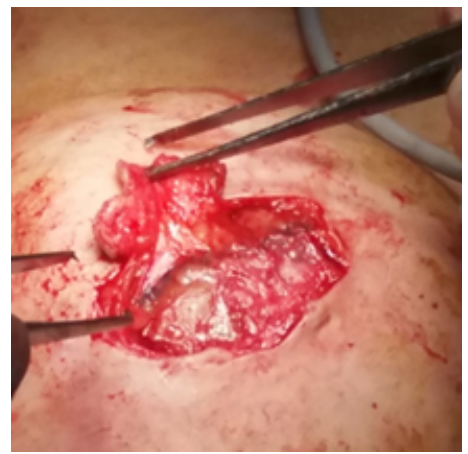

Fig.1. Synthetic mesh with cellular ingrowth within the pores and new tissue formation over the implant at 1 year after immediate breast reconstruction with implants and synthetic mesh support

\footnotetext{
* email: sorin.berbece@gmail.com; Phone: 0728889897
} 
The second - Type B mesh is a nonabsorbable mesh composed of polypropylene and coated with titanium, that has been approved for breast reconstructions in Europe since 2008 [11]. Similar to the first synthetic mesh used, it has a hydrophilic, lightweight structure, with competitive biocompatibility [2]. Unlike other synthetic meshes on the market, the titanium coating of these meshes reduces the inflammatory response and subsequent mesh contraction [12]. It can be found in 2 forms: as a light mesh with a tensile strength of 61 Newton at $35 \mathrm{~g} / \mathrm{m}^{2}$ and as an extralight mesh with a tensile strength of $37 \mathrm{~N}$ at $16 \mathrm{~g} / \mathrm{m}^{2}$ [2].

The third material - Type $\mathrm{C}$ is an absorbable synthetic mesh composed of fast degradable copolymer of lactide, glycolide and trimethylene carbonate which is degraded in the first 6 months after surgery, and a slow degradable copolymer of lactide and trymetylene carbonate which is kept in place for further support up to 3 years after implantation [2]. The absorption process of these mesh is possible due to the hydrolisis process that eventually eliminates the copolymers through normal metabolic pathways [13].

\section{Experimental part}

A prospective, nonrandomized study was performed evaluating 12 patients with immediate implant-based breast reconstruction with synthetic mesh support. The patients enrolled in the study between 2014 and 2017 underwent nipple-sparing or skin-sparing mastectomy followed by unilateral or bilateral breast reconstruction procedures. All patients that met these criteria were considered, including 2 patients with neoadjuvant chemotherapy completed before surgery, 1 patient with noninsulin dependent type II diabetes and 4 patients that were active smokers. Mutations of the BRCA1/BRCA2 gene were found in 2 patients. The only exclusion criteria involved patients who were considered for radiotherapy adjuvant treatment postoperatively. As radiotherapy is a compelling risk factor for immediate implant-based breast reconstruction with or without synthethic mesh support, patients expected to follow this treatment can benefitmore from alternative reconstructive procedures and implantbased breast reconstruction is best to be avoided.

The volume of the breast implants used for reconstruction ranged from $320 \mathrm{cc}$ to $550 \mathrm{cc}$ and depended on intraoperative aspects and individual anatomy of each patient. All synthetic meshes used measured $10 \times 15 \mathrm{~cm}$. The age of the patients at the time of surgery ranged from 32 to 56 years, leading to an average age of 43 years. Followup period of patients ranged from 2 to 24 months and included all patients that participated in the study.

\section{Surgical technique \\ In immediate implant-based breastreconstructions, the pectoral muscle has a very important role of coverage and support for the breast implant, which is usually placed in the rigurously dissected subpectoral pocket. The downfall of this procedure is that generally, the pectoral muscle can only cover the upper $2 / 3$ and medial quadrant of the breast implant, overlooking the inferior and lateral parts. Traditionally, this downfall was solved by mobilizing the serratus anterior muscle and anterior fascia of the rectus muscle, which concluded to more trauma to the native tissue, poor delineation of the inframammary fold and an unnatural contour of the reconstructed breastmound with an ascended, dilatated upper pole. A better technique to fix the pectoral muscle to the inframmary fold could be achieved by supporting it with a synthetic mesh.}

After completion of the nipple-sparing or skin-sparing mastectomy and creation of the subpectoral pocket, a synthetic mesh is fixed with sutures to the thoracic wall, $1.5 \mathrm{~cm}$ below the inframammary fold and laterally on the anterior axillary fold. After the implant is placed in the designed retropectoral pocket, the synthetic mesh is spread over the implant and sutured cranially to the inferolateral margin of the pectoral muscle and in certain cases to the anterior margin of the serratus anterior muscle. The implant is thus accommodated in a fully closed pocket that assures its position, support and coverage while granting better aesthethic and contour to the reconstructed breast. Antibiotics are recommended for the perioperative period and usually continued as long as the drains are kept in place. This surgical technique is well tolerated by patients and by virtue of the pores present in the synthetic mesh the cells can grow within the pores and thus generate new tissue to cover the implant.

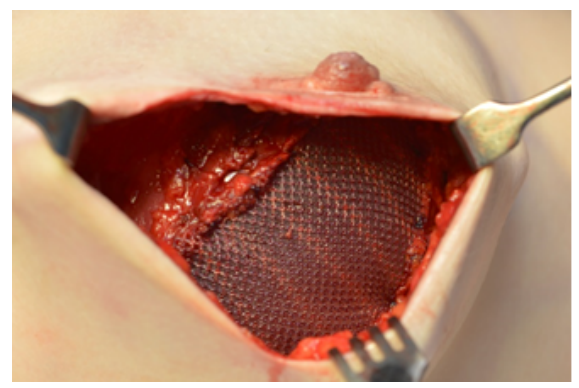

Fig. 2. Type $A$ synthetic mesh was used to cover the inferior and lateral aspect of the implant and obtain a fully closed pocket within which the implant was well positioned

\section{Results and discussions}

Our study population included 12 patients with immediate breast reconstruction - 9 of the patients had bilateral breast reconstruction and the other 3 patients had unilateral breast reconstruction. Generally, the surgical interventions were performed following malignant conditions - 10 patients had unilateral breast cancer and 1 patient had bilateral breast cancer. As a prophylactic measure, 8 of the patients with unilateral breast cancer, from which 2 patients diagnosed with BRCA1/BRCA2 gene mutation, chose to also have simple mastectomy of the contralateral breast. Another patient with a history of unilateral breast carcinoma in situ opted for bilateral prophylactic mastectomy. All patients had nipple-sparing or skin-sparing mastectomy followed by immediate breast reconstruction with definitive breast implants and synthetic mesh. Moreover, for 3 patients who presented with large volume breasts and significant ptosis, a plus to the synthetic mesh support was the use of a dermal sling created from the de-epithelized inferior postmastectomy flap.

We experienced no intraoperative complications. In the postoperative period we were not faced with any reconstructive failure like infection, implant loss, rejection or need for surgical reintervention. Postoperative complications included two patients with seroma that required puncture for drainage, one mild wound dehiscence at a vegetarian patient, one partial nipple necrosis in a

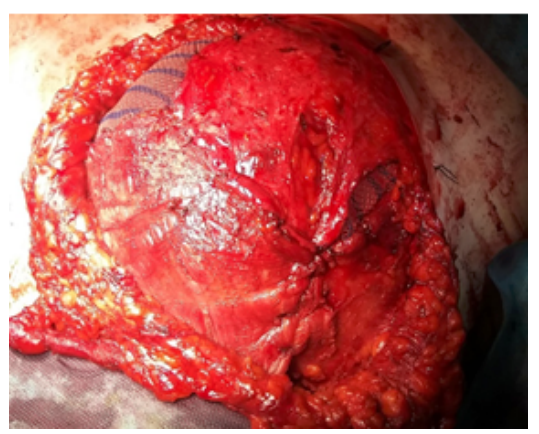

Fig. 3. Aspect of breast reconstruction with implant, synthetic mesh support associated with de-epithelized inferior postmastectomy flap (dermal sling) 
smoker patient and one case of volume asymmetry between the reconstructed breasts caused by contrasting thickness of the mastectomy flaps. Other complications involved a patient with a mild localized erythema which regressed spontaneously after 12 days and a patient with implant rippling who underwent remodelling by lipofilling 6 months after the reconstruction. All patients that participated in the study were able to receive undelayed adjuvant treatment after the reconstructive procedure. Furthermore, for the 7 patients with significant comorbidities ( 2 patients with neoadjuvant chemotherapy, 1 patient with noninsulin dependent type II diabetes and 4 smoker patients), final results were similar to those registered for the other patients involved in the study.

The main target of a breast reconstruction is oncological safety. If this target is accomplished, then we can analyze the aesthetic outcome that was obtained. After a medium follow-up of 13 months, there were no local or distant recurrences. In addition, aesthetic outcome and patient satisfaction were remarkable for all patients enrolled in the study, with no adverse reactions and good integration of the synthetic mesh within the implant's capsule.

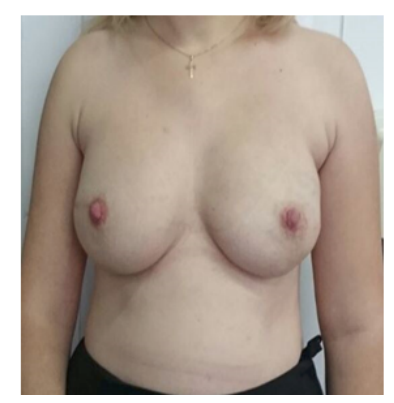

Fig. 4. 1 year follow-up result after bilateral prophylactic mastectomy and bilateral reconstruction with implants (395 cc) associated with synthetic mesh support (frontal view)

The aesthetic outcome of an immediate implant-based reconstruction with synthetic mesh support was demonstrated at the patient presented below (1 year follow-up). The 32 years old patient had a bilateral prophylactic mastectomy for in situ carcinoma of the right breast followed by bilateral breast reconstruction with cohesive gel implants (395 cc) and synthetic mesh support.

As you can observe, beautiful shape and form was obtained for the reconstructed breast, with equally distributed volume bilaterally, nice contour from the upper chest wall to the peak of the nipple and areola and a wellcentered nipple-arreolar complex at the maximum point of projection on the chest wall. The synthetic mesh support granted a natural contour and good delineation of the inframmary fold, supported the skin flaps and lower pole offering good stability and projection to the reconstructed breasts.

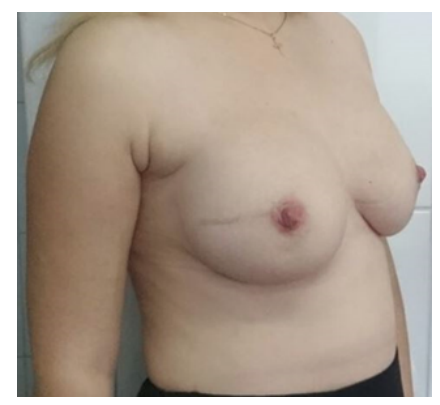

Fig. 5. 1 year follow-up result after bilateral prophylactic mastectomy and bilateral reconstruction with implants (395 cc) associated with synthetic mesh support (side view)

If we decide for a strictly submuscular pocket to accomodate the implant, we might be faced with the risk of a high-riding implant of restricted volume, unfavourable projection and shape of the breast mound, with unnatural ptosis and poor inframammary delineation. Advantages of using a synthetic mesh include the implant's position and control, but also the possibility for increased volume of the implant, better defined inframmary and lateral folds, while the need for contralateral mastopexy decreases considerably. Results obtained so far, lead us to believe that synthetic meshes can offer better aesthetic outcomes for immediate breast reconstructions, at lower costs and with fewer complications [8]. Synthetic meshes are nonallergenic and easily accesible at relatively low costs. Moreover, due to their low inflammatory reaction and highresistance to biofilm formation, it has been hypothesized that their use might decrease the incidence of inflammation, infection, seroma and capsular contracture in implant-based breast reconstructions [14].

Published data until date has not concluded to any noticeable differences between the long-term results of these synthetic meshes [15]. Moreover, complication rates for breast reconstructions with or without synthetic meshes are quite similar, ranging from $17.7-29 \%$ for breast reconstructions with synthetic meshes to $15 \%$ for breast reconstructions without synthetic meshes. Furthermore, publications comparing synthetic meshes to biological matrices noted that seroma rates of $1.8-4.8 \%$ in the context of synthetic meshes use are much lower than those found for biological matrices use (0-32\%), probably due to the difference in surface that these two materials withstand $[2,15-20]$. The smooth surface of biological matrices increases the risk of seroma between the matrice and the subcutaneous tissue overlying it, while the rough surface of synthetic meshes interacts better with the subcutaneous tissue, thus reducing the risk of liquid formation and seroma [2].

Another important factor to consider when deciding upon biological matrices or synthetic meshes is the difference in thickness between these two materials. Based on this factor, synthetic meshes are predominantely used in immediate-breast reconstruction procedures where thick, well-vascularized mastectomy flaps permit it, and biological matrices are better used in delayed breast reconstruction procedures which require adequate softtissue substitute [2].

The aim of our analyze of synthetic meshes was to present additional solutions for IBBR surgery and to better understand the implications of synthetic meshes in the postoperative complications. Although these materials bring about significant value in the ever growing field of breastreconstruction surgery, with high patient satisfaction, good aesthetic results and low complication rates, it is necessary to further analyze the long-term results that these materials produce.

\section{Conclusions}

Synthetic meshes appear promising for breast reconstruction patients that require both a better stability of the implant and a better countour of the reconstructed breast. Results to date have been encouraging, with a low complication rate and excellent subjective patient satisfaction and aesthetic results. Continued follow-up is planned to evaluate long-term results.

Accordingly, we consider synthetic meshes a step further to the future of breast reconstruction and believe they will bring significant value in the course of treatment for patients undergoing implant-based breast reconstructive surgery

Disclosure: The authors have no financial interest to declare in relation to the content of this article.

\section{References}

1.SCOTT S., MESHABI A., Implant-based reconstruction, Clin Plast Surg, 34(1), 2007, p.63-73

2.MAX D., FARIDI A., Biological matrices and synthetic meshes used in implant-based breast reconstruction, Geburtshilfe Frauenheilkd, 73(11), 2013, p.1100-1106. 
3.BAHMAN G., ELOF E., JOHN P., KEVIN C., JOSEPH D., ARUN G., BRIAN K., PETER R., Plastic Surgery. Indications and Practice, Edition 1, Saunders Elsevier, Amsterdam, 2009, p.264

4.CICERO U., MARIO R., Oncoplastic and reconstructive surgery of the breast, Edition 1, Springer-Verlag, Italy, 2013, p. $235-253$

5.HOLTON L., KIM D., SILVERMAN R., Human acellular dermal matrix for repair of abdominal wall defects: review of clinical experience and experimental data, J Long Term Eff Med Implants, 15, 2005, p.547558

6.EPPLEY B., Revascularization of acellular human dermis (AlloDerm) in subcutaneous implantation, J Aesthetic Surg, 21, 2000, p.291-295

7.OREN T., RICHARD R., DANIEL M., BARBARA S., WILLIAM A., Beyond biologics: absorbable mesh as a low-cost, low-complication sling for implant-based breast reconstruction, Plastic and Reconstructive Surgery, Volume 133, Issue 2, 2014, p. 90e-99e

8.MEYER G., MICKAEL T., THOMAS P., THANH L., ALI M., BADWI E., BRIGITTE P., Risks and benefits of using an absorbable mesh in onestage immediate breast reconstruction: a comparative study, Plastic and Reconstructive Surgery, Volume 135, Issue 3, 2015, p. 498e-507e 9.Seragyn $\mathrm{Br}$ - Mesh-assisted reconstruction of the female breast, http://www.serag-wiessner.de/en/ (accessed online on 03.03.2017) 10.MAX D., GERBER B., REIMER T., Biokompatibilität synthetischer und biologischer Netze im Rahmen der implantatgestützen Brustrekonstruktion, Posterbeitrag, Senologiekongress, Munchen, 2013

11.MAX D., PAEPKE S., ZWIEFEL K., DIETERICH H., BLOHMER J ., FARIDI A., KLEIN E., GERBER B., Implant-based breast reconstruction using a titanium-coated polypropylene mesh (TiLOOP Bra): a multicenter study of 231 cases, Plastic and Reconstrive Surgery, 132(1), 2013, p.8e-19e
12.SCHEIDBACH H., TANNAPFEL A., SCHMIDT U., Influence of titanium coating on the biocompatibility of a heavyw eight polypropylene mesh. An animal experimental model, Eur Surg Res,36, 2004, p.313-317 13. HILTON B., JEFFREY G., The use of synthetic mesh in reconstructive, revision and cosmetic breast surgery, Aesthetic Plastic Surgery, 37 (5), 2013, p. 914-921

14.MELANIE M., VICTOR W., EMILY N., MICHAEL L., GEOFFREY G., The foreign body response: at the interface of surgery and bioengineering, Plastic \& Reconstructive Surgery, Volume 135, Issue 5, 2015, p. 1489-1498

15.PETERSEN A., EFTEKHARI A., DAMSGAARD T., Immediate breast reconstruction: a retrospective study with emphasis on complications and risk factors, J Plast Surg Hand Surg, 46, 2012, p.344-348

16.IBRAHIM A., AYENI O., HUGHES K., Acellular dermal matrices in breast surgery: a comprehensive review, Ann Plast Surg, 70, 2013, p.732-738

17.CHUN Y., VERMA K., ROSEN H.,Implant-based breastreconstruction using acellular dermal matrix and the risk of postoperative complications, Plast Reconstr Surg., 125, 2010, p.429-436

18.WEICHMAN K., WILSON S., WEINSTEIN A., The use of acellular dermal matrix in immediate two-stage tissue expander breast reconstruction, Plast Reconstr Surg, 129, 2012, p.1049-1058

19.PELED A., FOSTER R., GARWOODE., The effects of acellular dermal matrix in expander-implant breast reconstruction after total skinsparing mastectomy: results of a prospective practice improvement study, Plast Reconstr Surg., 129, 2012, p.901e-908e

20.LUPASCU URSULESCU C.,URSARU M., CIOBANU D., NEGRU D., LUPASCU C. - Dystrophic Large Calcification in Breast Cancer, Rev. Chim.(Bucharest), 68, no.5, 2017, p. 1143

Manuscript received: 12.02 .2017 